\title{
RESEARCH
}

Open Access

\section{Impact of industrial production system parameters on chicken microbiomes: mechanisms to improve performance and reduce Campylobacter}

\author{
Aaron McKenna ${ }^{1,4 \dagger}$, Umer Zeeshan ljaz ${ }^{2 \dagger}$, Carmel Kelly ${ }^{3}$, Mark Linton ${ }^{3}$, William T. Sloan ${ }^{2}$, Brian D. Green ${ }^{4}$, \\ Ursula Lavery ${ }^{1}$, Nick Dorrell ${ }^{5}$, Brendan W. Wren ${ }^{5}$, Anne Richmond ${ }^{1}$, Nicolae Corcionivoschi ${ }^{3^{*+}}$ and \\ Ozan Gundogdu ${ }^{5^{*+}}$ (1)
}

\begin{abstract}
Background: The factors affecting host-pathogen ecology in terms of the microbiome remain poorly studied. Chickens are a key source of protein with gut health heavily dependent on the complex microbiome which has key roles in nutrient assimilation and vitamin and amino acid biosynthesis. The chicken gut microbiome may be influenced by extrinsic production system parameters such as Placement Birds $/ \mathrm{m}^{2}$ (stocking density), feed type and additives. Such parameters, in addition to on-farm biosecurity may influence performance and also pathogenic bacterial numbers such as Campylobacter. In this study, three different production systems 'Normal' (N), 'Higher Welfare' (HW) and 'Omega-3 Higher Welfare' $(O)$ were investigated in an industrial farm environment at day 7 and day 30 with a range of extrinsic parameters correlating performance with microbial dynamics and Campylobacter presence.

Results: Our data identified production system $\mathrm{N}$ as significantly dissimilar from production systems HW and O when comparing the prevalence of genera. An increase in Placement Birds $/ \mathrm{m}^{2}$ density led to a decrease in environmental pressure influencing the microbial community structure. Prevalence of genera, such as Eisenbergiella within HW and $\mathrm{O}$, and likewise Alistipes within N were representative. These genera have roles directly relating to energy metabolism, amino acid, nucleotide and short chain fatty acid (SCFA) utilisation. Thus, an association exists between consistent and differentiating parameters of the production systems that affect feed utilisation, leading to competitive exclusion of genera based on competition for nutrients and other factors. Campylobacter was identified within specific production system and presence was linked with the increased diversity and increased environmental pressure on microbial community structure. Addition of (Continued on next page)
\end{abstract}

\footnotetext{
*Correspondence: Nicolae.Corcionivoschi@afbini.gov.uk;

Ozan.Gundogdu@lshtm.ac.uk

${ }^{\dagger}$ Aaron McKenna and Umer Zeeshan ljaz are joint first authors. Nicolae Corcionivoschi and Ozan Gundogdu jointly directed this work.

${ }^{3}$ Food Microbiology Unit, Agri-Food and Biosciences Institute, Newforge Lane, Belfast BT9 5PX, UK

${ }^{5}$ Faculty of Infectious \& Tropical Diseases, London School of Hygiene and Tropical Medicine, Keppel Street, London WC1E 7HT, UK

Full list of author information is available at the end of the article
}

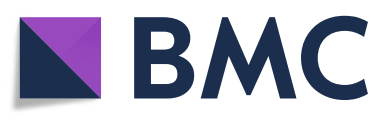

( ) The Author(s). 2020 Open Access This article is licensed under a Creative Commons Attribution 4.0 International License, which permits use, sharing, adaptation, distribution and reproduction in any medium or format, as long as you give appropriate credit to the original author(s) and the source, provide a link to the Creative Commons licence, and indicate if changes were made. The images or other third party material in this article are included in the article's Creative Commons licence, unless indicated otherwise in a credit line to the material. If material is not included in the article's Creative Commons licence and your intended use is not permitted by statutory regulation or exceeds the permitted use, you will need to obtain permission directly from the copyright holder. To view a copy of this licence, visit http://creativecommons.org/licenses/by/4.0/. The Creative Commons Public Domain Dedication waiver (http://creativecommons.org/publicdomain/zero/1.0/) applies to the data made available in this article, unless otherwise stated in a credit line to the data. 
(Continued from previous page)

Omega-3 though did alter prevalence of specific genera, in our analysis did not differentiate itself from HW production system. However, Omega-3 was linked with a positive impact on weight gain.

Conclusions: Overall, our results show that microbial communities in different industrial production systems are deterministic in elucidating the underlying biological confounders, and these recommendations are transferable to farm practices and diet manipulation leading to improved performance and better intervention strategies against Campylobacter within the food chain.

Keywords: Chicken, Microbiome, Campylobacter, Production systems, Environmental filtering, Phylogenetic signal, Competitive exclusion, Diversity

\section{Background}

Chickens are a key source of protein for humans where poultry production is predicted to produce approximately 130 million tons of chicken meat in 2020 [1, 2]. Sustainable poultry practices are needed to help maintain an adequate supply of poultry products for the increasing human population without compromising the chicken or human health [3]. Selective breeding programmes has resulted in chickens that efficiently convert food into body mass, as defined by feed conversion ratios (FCR) [4]. The fundamental component needed to ensure an efficient poultry production is highly dependent in having an optimised nutrition and production setup [1].

Production systems vary immensely between countries, businesses and at farm level. Certain parameters such as Placement Birds $/ \mathrm{m}^{2}$ (stocking density), Protein_perc_ration (protein percentage within ration) and Energy_of_ration (energy content) in relation to the feed are key determinants that if varied, may directly influence chicken microbial community structure, thus impacting performance and potentially reduction of pathogenic bacteria. Chicken diets are typically formulated to enhance production efficiency. However, some diets are formulated to enhance human health, such as diets containing Omega-3 polyunsaturated fatty acids (PUFA's) [5]. There is anecdotal evidence at farm level suggesting that this enrichment has potential to improve chicken gut health and performance or reduce pathogen colonisation.

Many bacterial species found within the microbiome of farmed animals can potentially be considered pathogens and detrimental to human health. One of these pathogens is Campylobacter which is the leading cause of human foodborne bacterial gastroenteritis causing bloody diarrhoea, fever and abdominal pains in humans and can also cause post infectious sequelae such as Guillain-Barré syndrome which is a potentially fatal paralytic autoimmune illness [6]. Campylobacter jejuni (predominant species causing infection to humans) colonises chicken caeca with relatively high numbers $\left(>10^{9} \mathrm{CFU} / \mathrm{g}\right)$ and can be pathogenic to the chicken, with this dependent on the genetics of host and strain of infection [7-10]. Although there exist many intervention studies, in an industrial farm environment, we currently do not understand why we typically see Campylobacter at approximately two weeks into the chicken life cycle [11-13]. The natural growth and flux of the gut microbiome may have a role to play [14]. This is further convoluted by the fact that there are very few studies on how chicken diet impacts Campylobacter presence within the chicken gut microbiome, particularly from within an industrial farm environment.

Chicken performance and gut health is heavily dependent on the complex gut microbial community which plays a role in nutrient assimilation, vitamin and amino acid biosynthesis and prevention of pathogen colonisation [15-18]. The microbiota is responsible for hydrolysing indigestible carbohydrates and polysaccharides allowing further fermentation by other members of the gut ecosystem that produce short chain fatty acid (SCFA), in turn allowing utilisation by the host [1]. The relationship between gut microbiota, chicken health and performance represents a tripartite that has been under the scrutiny of the research community with the prospect of improving the efficiency of current microbiome manipulation strategies $[19,20]$. As an example, a xylanase gene from chicken caecum has been isolated and overexpressed, potentially leading to development of new feed additives for industrial application [21]. Our understanding of diet and its impact on the intestinal microbiota is still nascent and requires further exploration.

In the present study, we aim to build on our previous research where we performed a comprehensive analysis of the chicken caecal microbiome from days 3 to 35 investigating the driving forces of bacterial dynamics over time, and how this relates to Campylobacter appearance within a natural habitat setting [14]. Microbial variation over time was heavily influenced by the diet, where significant shifts in bacterial composition were observed [14]. The factors affecting host-pathogen ecology in terms of microbial community structure remain poorly 
studied at an industrial farm level. Therefore, extending our previous work and in view of the above ambitions, in this study, three different industrial production systems, namely, 'Normal' (N), 'Higher Welfare' (HW) and 'Omega-3 Higher Welfare' $(\mathrm{O})$ were investigated at day 7 and day 30, along with extrinsic parameters (which were not available in our previous study) to ascertain mechanisms on improving the overall performance of chickens and also elucidating the role of microbial community dynamics on revealing Campylobacter.

\section{Materials and methods}

\section{Ethics statement}

Euthanasia of birds was carried out as previously described [14] under veterinary supervision alongside routine veterinary diagnostic inspection and after consultation and approval from the ethical committee within Moy Park. Briefly, euthanasia was performed either by dislocation of the neck or by dislocation of the neck followed by anaesthesia with isoflurane depending on the weight of the bird.

\section{Experimental design, broilers and sample collection}

Chickens reared under three different industrial growing regimes, 'Normal' (N), 'Higher Welfare' (HW) and 'Omega-3 Higher Welfare' $(\mathrm{O})$, were sourced from three different contract farms supplying chicken to Moy Park (39 Seagoe Industrial Estate, Portadown, Craigavon, Co. Armagh, BT63 5QE, UK).

Although the three production systems differ in receiving chicks from multiple flocks, our analysis suggested that they had no bearing on microbial community structure at finer level (Fig. 3 and discussion later) although some parameters were implicated as significant in PERMANOVA analysis. All chicks were Ross 308 as hatched (AH) and were supplied from the same Moy Park hatchery to all three farms on 11 October 2018. All birds were grown in typical industrial poultry houses and were raised on a four-stage diet made up of a starter, grower, finisher and withdrawal ration; however, composition of these diets differed across the three growing regimes. For farm N, birds were offered standard starter ration from days 0 to 11 , standard grower ration from days 11 to 22 and standard finisher from day 22 to day 34 before moving to a standard withdrawal ration prior to slaughter. For farm HW, birds were offered higher welfare starter ration from days 0 to 11 , higher welfare grower ration from days 11 to 23 and higher welfare finisher from day 23 to day 31 before moving to a higher welfare withdrawal ration prior to slaughter. For farm $\mathrm{O}$, birds were offered higher welfare starter ration from days 0 to 11 , higher welfare grower ration from days 11 to 20 and Omega-3 finisher ration from day 20 to day 30 before moving to an Omega-3 withdrawal ration prior to slaughter. Both Omega-3 finisher rations are identical to HW equivalent ration, but with addition of an Omega-3 premix produced by Devenish Nutrition Ltd (Belfast, UK) and added at the feed mill. At day 7, 19 chickens and at day 30, 10 chickens were randomly removed from the same single poultry house on each of the three farms.

\section{Campylobacter isolation and identification}

The contents of a pair of caeca were transferred into a sterile stomacher bag $(\sim 10 \mathrm{~g} \pm 1 \mathrm{~g})$, diluted with maximum recovery diluent (MRD) (Oxoid, UK) buffer to make a $1 / 10$ dilution and stomached at $260 \mathrm{rpm}$ for 1 min. Further decimal dilutions were carried out in MRD to give a range of dilutions suitable to achieve countable plates. One hundred micro litres of the original suspension $\left(10^{-1}\right.$ dilution) was inoculated onto duplicate plates of modified charcoal cefoperazone deoycholate agar (mCCDA) (Oxoid, UK). The inoculum was spread uniformly over the surface of the agar plate with a sterile spreader until fully absorbed. This spread plating was repeated for all the other decimal dilutions. When low numbers of Campylobacter were expected, the limit of detection was increased by also spread plating $1 \mathrm{ml}$ of the original suspension. The 1 $\mathrm{ml}$ inoculum was inoculated over three plates. The plates were incubated in a microaerophilic atmosphere (5\% oxygen, $10 \%$ carbon dioxide and $85 \%$ nitrogen) at $41.5^{\circ} \mathrm{C}$ and examined after $44 \pm 4 \mathrm{~h}$ for typical colonies of Campylobacter spp. Plates containing less than 300 colonies were counted. Colonies were considered typical if they were greyish (often with a metallic sheen), flat and moist, with a tendency to spread. Campylobacter numbers were expressed as $\mathrm{CFU} / \mathrm{g}$ caecal content. Genomic DNA (gDNA) was also extracted from chicken caeca for $16 \mathrm{~S}$ metagenomics experiments.

\section{Poultry growth and performance measurements}

Performance parameters on per flock basis were recorded in line with typical industrial practices. Weight Gain_per_Day, Feed_Conversion_Ratio, Total_Mortality_percentage, Energy_of_Ration, Protein_perc_ration, Water_Consumption_per_Bird, Age_At_Thin, Age_at Total_Depopulation, PMI_Rejects_percentage, Hockmark_percentage, Pododermatitis_percentage, log_CFU_ per_g_Campylobacter and European Poultry Efficiency Factor (EPEF) were all captured (Supplementary S13). These variables were then correlated with the microbial community's composition in various statistical analyses.

\section{DNA extraction, 16S rRNA amplification and sequencing}

Caecal gDNA was extracted using QIAamp DNA Stool Mini Kit according to the manufacturer's instructions and stored at $-20^{\circ} \mathrm{C}$. $16 \mathrm{~S}$ metagenomic sequencing library construction was performed using Illumina 
guidelines (Illumina, USA). The $16 \mathrm{~S}$ ribosomal primers used were V3 (tcgtcggcagcgtcagatgtgtataagagacagcctacgggnggcwgcag) and V4 (gtctcgtgggctcggagatgtgtataagagacaggactachvgggtatctaatcc) [22, 23]. A second PCR step was performed based on the manufacturer's guidelines to attach dual indices and Illumina sequencing adapters using the Nextera XT Index kit. Sequencing was performed on Illumina MiSeq at LSHTM using a v3 300 bp paired-end kit.

\section{Bioinformatics and statistical analysis}

Details are described in the supplementary methods (Supplementary S12).

\section{Results}

Diversity patterns representative of the production systems At alpha-diversity level, to investigate how diversity within the samples were influenced by the production system, both richness and Shannon entropy were calculated for day 7 and day 30 samples from production systems N, HW and O. In the host microbiome, time has a significant effect on microbial richness with all production systems significantly increasing from day 7 to day 30 (Fig. 1a). For day 7, production system $\mathrm{N}$ displayed the highest microbial richness when compared to HW and O. At day 30, no statistical difference in terms of richness was identified between the three production systems. Beta diversity using Bray-Curtis was measured, along with these operational taxonomic units (OTUs) collated together at genera level with only top- 25 most abundant genera shown next to the PCoA diagram (Fig. 1b). At day 7, in terms of abundance counts, samples from production system $\mathrm{O}$ are far off from the clusters that contain samples from $\mathrm{N}$ and $\mathrm{HW}$, respectively. On the other hand, at day $30, \mathrm{~N}$ seems to have a different community structure as compared to $\mathrm{N}$ and HW production systems, respectively. The breakdowns of taxa going from finer (OTUs), up coarser levels (family, class, phylum, etc.), are shown in Supplementary S3. Note, we are only considering the top- 25 most abundant taxa identified at different taxonomic levels. Of interest, clear differences were observed using visual cues when comparing production system $\mathrm{N}$ at day 30 to $\mathrm{HW}$ and $\mathrm{O}$, where genera Bacteroides and Alistipes were present in production system $\mathrm{N}$ at higher abundances.

Ecological drivers of microbial community were explored observing the clustering in the phylogenetic tree of the OTUs and utilising phylogenetic alpha diversity measures such as NRI/NTI. Positive NRI/NTI values indicate strong phylogenetic clustering (driven by environmental filtering), whereas reduced values represent phylogenetic overdispersion (environment has little or no role to play). Here, using NRI and NTI, we can observe an increase in $\mathrm{N}, \mathrm{HW}$ and $\mathrm{O}$ samples respectively from day 7 to day 30 . Since chicken caeca are already a constrained environment to begin with (as opposed to real environmental datasets), the values $<0^{1}$ (traditionally this implies stochasticity) may not be feasible to ascertain randomness/stochasticity/competitive exclusion principle, and hence values should be taken relatively with an increasing value implying increasing host environmental pressure (in the immediate case it is the environment within a chicken, whilst extrinsic environmental factors such as surroundings where chickens are confined and their diet may also have a role to play). Figure 1a corroborates our findings from our previous longitudinal study [14].

\section{Key drivers of microbial community structure variation in terms of beta diversity}

Next, we wanted to explore what drives the beta diversity amongst different categories (production system) and time. In this regard, we employed the 'BVSTEP' routine [24] which reduces the complexity of microbiome datasets by getting rid of any features from the abundance table that do not contribute to beta diversity between samples. Briefly, the procedure calculates pair-wise Bray-Curtis distances between samples using all the features (ASVs). It then records this as a ground truth, and in the second step permutes through the combinations of ASVs, calculates the beta diversity distances over and over again using these permutations, and homes on to minimal subsets of these ASVs where the beta diversity is roughly conserved against the ground truth by maximising the correlation (Supplementary S1). The resulting members of the subset(s) are then tested to see if (a) they still explain the variability between multiple groups (PERMANOVA analysis on these reduced subsets) and (b) if some of the members of these subsets have discriminating abundances between multiple groups (DESeq2, differential heat trees and MINT analysis). By using this approach, we are able to highlight the important members of microbial community. It should be noted that we have employed several analytical techniques to mask out biases associated with their underlying mathematical formulation and to give a consensus agreement on what stands out consistently. The parameterisation of these techniques are as follows: (i) DESeq2 was used with adjusted $p$ value significance cut-off of 0.05 and $\log 2$ fold change (Supplementary S11), (ii) differential heat tree analysis was performed on

\footnotetext{
${ }^{1}$ It should be noted that NRI reflects phylogenetic clustering in a broad sense (whole phylogenetic tree) with lower values representing evenly spread community. On the other hand, NTI focuses more on the tips of the tree with positive values of NTI indicating that species co-occur with more closely related species than expected, and lower values indicating that closely related species do not co-occur by chance
} 
A) Alpha Diversity Measures
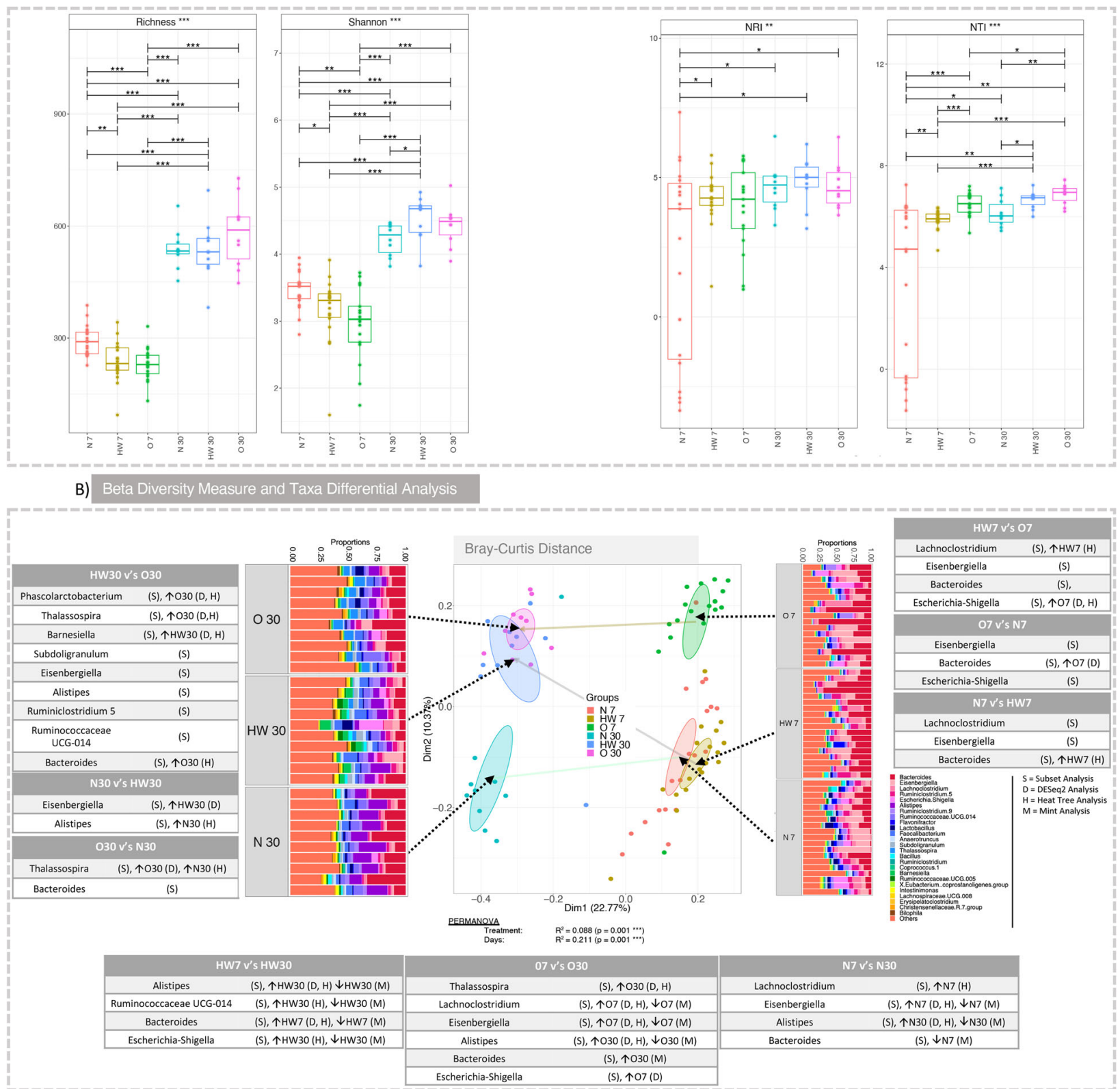

Fig. 1 Microbial diversity and community structure. a Alpha diversity (Richness and Shannon entropy) and environmental filtering (NRI/NTI) measures respectively. Lines (a) connect two categories where the differences were significant (ANOVA) with ${ }^{*} P<0.05,{ }^{* *} P<0.01$, or ${ }^{* * *} p<0.001$. b Beta diversity using Bray-Curtis distance measure along with top-25 genera observed in all samples grouped by categories. The tables represent taxa that were found to be significant based on subset analysis (Supplementary S1), i.e. those genera selected in the subsets that explain roughly the same distance between samples as all the genera. Additionally, if the taxa were found to be differentially expressed based on other analyses, such as DESeq2 (Supplementary S11), MINT (Supplementary S2) and differential heat tree (Fig. 2), the categories they up- and downregulated are represented with corresponding up and down arrows. For example, in HW30 vs $\mathrm{O} 30$ comparison, '(S), O30 (D, H)' for Phascolarctobacterium should be read as selected in subset analysis: (S) and upregulated in O30 according to both DESeq2 and Differential Tree: O30 (D, H)

proportional normalisation to find clades that were differentially expressed (Fig. 2b) and (iii) MINT analysis was performed to see if we could consolidate the genera that have simultaneous discriminatory power at both spatial (production system N, HW and $\mathrm{O}$ ) and temporal scales (day 7 and day 30) (Supplementary S2). MINT gives an overall discriminatory power considering all sources of variations including the studies (production system) and temporal scales (days). Whilst MINT gives an overall discriminatory power considering all sources of variations 


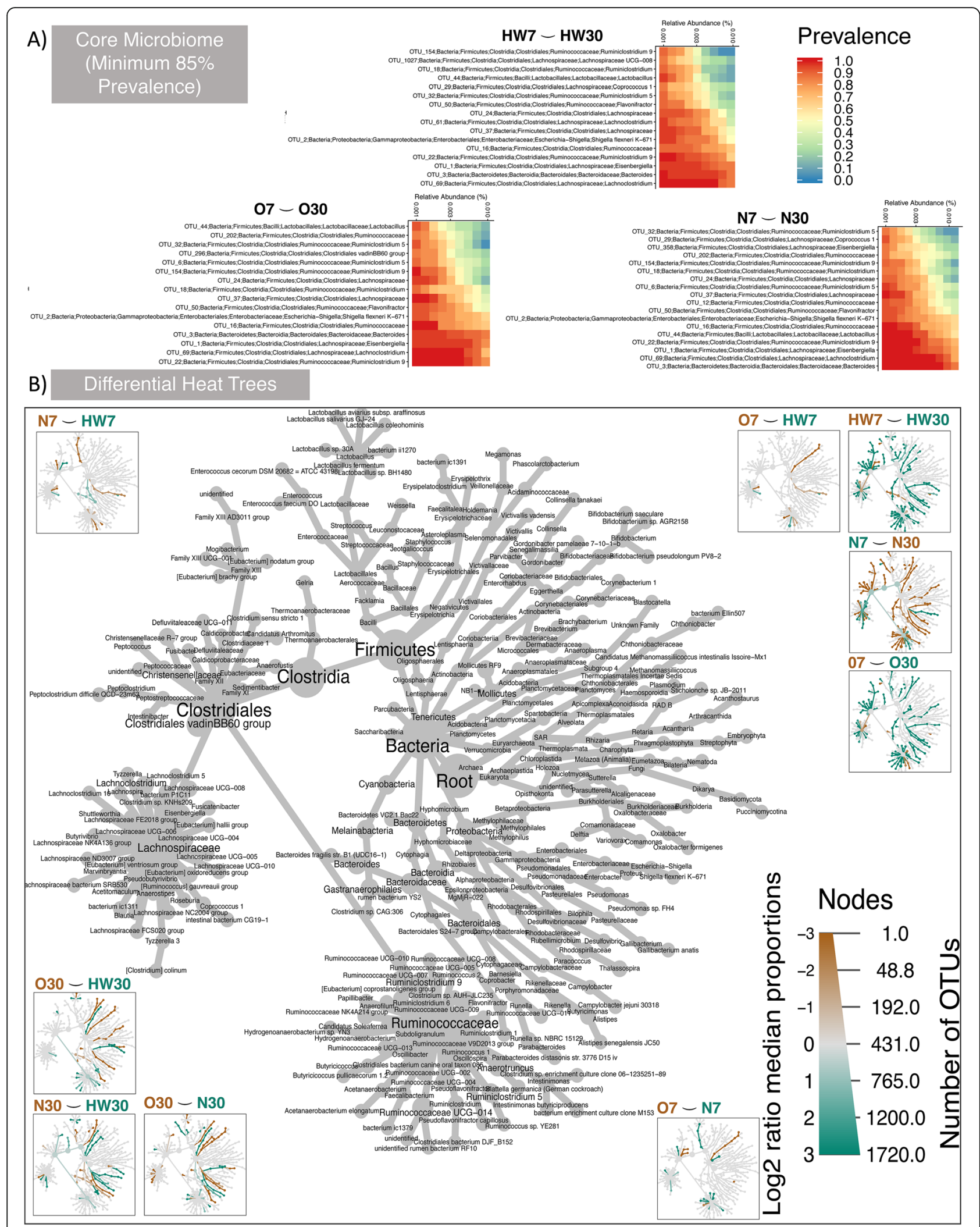

Fig. 2 (See legend on next page.) 
(See figure on previous page.)

Fig. 2 Taxa that persist and those that are differentially abundant. a Core microbiome analyses that persist in 85\% of the samples for different production systems (day 7 and day 30). In the heat maps, the OTUs are sorted by their abundances with those on the top being low abundant, whereas those at the bottom are highly abundant. b Differential heat tree with taxonomy key given in the middle, and the branches where they are upregulated are coloured according to their respective categories shown on top of each subpanel

including the studies (production system) and temporal scales (days), it may not be very efficient in terms of picking out the key features, as our results show marked disagreement with DESeq2 and differential heat trees, nonetheless, where it has worked, provided further credence to the underlying patterns being as realistic as they can be. To aid interpretation, all up/downregulated taxa in terms of abundances are annotated with up and down arrows in Figure 1 according to which analysis they were selected in and with taxa from subset analysis as the seeding point.

For production system HW, Alistipes, Ruminococcaceae UCG-014 and Escherichia-Shigella were significant genera increasing from day 7 to day 30. Bacteroides displayed significant increase at day 7 (decreasing at day 30) using DESeq2 and Heat Tree. For production system $\mathrm{O}$, Thalassospira, Alistipes and Bacteroides increased at day 30 (Bacteroides directionality was only observed with MINT analysis). Lachnoclostridium, Eisenbergiella and Escherichia-Shigella all increased at day 7 (decreasing at day 30). For production system N, Lachnoclostridium and Eisenbergiella increased at day 7 (decreasing at day 30). Bacteroides was identified as decreasing at day 7 (increasing at day 30; directionality was only observed with MINT analysis). In general, Alistipes was observed to increase consistently at day 30 for all production systems, whilst all other genera showed mixed trends. Although it should be noted that Alistipes was present at higher abundance at production system $\mathrm{N}$ as compared to others.

Key genera were identified when comparing production systems at day 7. For HW vs O comparison, Lachnoclostridium was identified as increased for HW. Escherichia-Shigella was identified increased for production system O. For $\mathrm{O}$ vs $\mathrm{N}$ comparison, Bacteroides was increased for $\mathrm{O}$. This was also replicated for HW when comparing to N. Key genera were identified when comparing production systems at day 30. For HW vs O comparison, Phascolarctobacterium, Thalassospira and Bacteroides were identified as increased for $\mathrm{O}$, whereas Barnesiella was increased for HW when comparing to O. Subdoligranulum, Eisenbergiella, Alistipes, Ruminiclostridium 5 and Ruminococcaceae UCG-014 were all identified as part of the subsets that explain beta diversity, although they were not implicated as discriminating in differential analyses. For $\mathrm{N}$ vs $\mathrm{HW}$ comparison at day 30, Eisenbergiella was increased for HW, and Alistipes was increased for N. For O vs N comparison at day 30, Bacteroides was observed with subset analysis alone. Thalassospira was observed, yet its discriminatory power was inconclusive.

Core microbiome where genera persist in $85 \%$ of the samples (something that is traditionally used to define the prevalence of core microbiome) for different production systems (day 7 and day 30) was assessed (Fig. 2). Genera identified include Bacteroides, Lachnoclostridium, Eisenbergiella, Ruminoclostridium 9, Lactobacillus, Ruminococcaceae, Shigella flexneri K-671, Flavonifractor, Ruminococcaceae, Lachnospiraceae, Ruminiclostridium 5, Ruminiclostridium, Coprococcus 1 and Ruminiclostridium 5 at varying level of abundance. In Fig. 2, OTUs are sorted by their abundances with those on the top being low abundant prevalent OTUs, whereas those at the bottom are highly abundant prevalent OTUs.

\section{Parameters deriving microbial community structure}

Analysis of parameters that had a significant effect on microbial diversity were assessed using PERMANOVA against performance parameters when using different dissimilarity measures on microbiome data (Table 1). Using $R^{2}$, if significant, to represent the variability explained in the community structure for Bray-Curtis distance, the parameter with the greatest impact was days, explaining $21.2 \%$ of the variation. Next, key parameters of interest were log_CFU_per_g_Campylobacter (4.1\% variability), Energy_of_Ration (4.0\% variability) and Protein_perc_ration ( $1.2 \%$ variability). Using both unweighted and weighted UniFrac as a beta diversity measure, the pattern was more or less similar with the same trends with days having the greatest impact on microbial diversity $(32.7 \%$ and $43.0 \%$ respectively). All other parameters had $R^{2}$ values at $1-5 \%$.

\section{Direction of influence for extrinsic parameters influencing key metrics for the microbiome}

Whilst PERMANOVA analyses show the extent of influence on microbiome structure in terms of variability, to obtain directions as to whether an increase or decrease in these parameters causes an increase or decrease in the properties of microbiome, we resorted to performing subset regressions on one-dimensional realisation of microbiome (alpha, beta diversity measures, etc.). These subset regressions permuted through all possible subsets of explanatory variables (extrinsic parameters considered in this study) by ranking them in terms of quantitative fit after performing cross-validation (Supplementary S4 to $\mathrm{S} 10$ and summarised in Fig. 3). Note that red and blue 
Table 1 Statistics for PERMANOVA against performance parameters when using different dissimilarity measures on microbiome data. $R^{2}$ represents the proportion of variability explained, for example, using 'Days' and 'Bray-Curtis' dissimilarity, the days explain 21.2\% variability in microbial community structure

\begin{tabular}{|c|c|c|c|c|c|c|}
\hline Predictors & Df & SumsOfSas & Meansqs & F.Model & $R^{2}$ & $p$ \\
\hline \multicolumn{7}{|l|}{ Bray-Curtis distance } \\
\hline Days & 1 & 4.9513 & 4.9513 & 26.8712 & $0.21163^{* * *}$ & $<0.001$ \\
\hline No. of parent flocks used & 1 & 1.065 & 1.065 & 5.7796 & $0.04552^{* * *}$ & $<0.001$ \\
\hline Birds placed & 1 & 1.0209 & 1.0209 & 5.5407 & $0.04364^{* * *}$ & $<0.001$ \\
\hline log CFU/g Campylobacter & 1 & 0.9606 & 0.9606 & 5.2131 & $0.04106^{* * *}$ & $<0.001$ \\
\hline Protein (\% of ration) & 1 & 0.2729 & 0.2729 & 1.4813 & 0.01167 & $<0.1$ \\
\hline Energy of ration (Kcal/lb AME) & 1 & 0.9375 & 0.9375 & 5.088 & $0.04007^{* * *}$ & $<0.001$ \\
\hline Residuals & 77 & 14.1881 & 0.1843 & 0.60642 & & \\
\hline Total & 83 & 23.3964 & 1 & & & \\
\hline \multicolumn{7}{|l|}{ Unweighted UniFrac distance } \\
\hline Days & 1 & 4.2369 & 4.2369 & 46.886 & $0.32727^{* * *}$ & $<0.001$ \\
\hline No. of parent flocks used & 1 & 0.3242 & 0.3242 & 3.588 & $0.02504^{* *}$ & $<0.01$ \\
\hline Birds placed & 1 & 0.6806 & 0.6806 & 7.532 & $0.05258^{* * *}$ & $<0.001$ \\
\hline log CFU/g Campylobacter & 1 & 0.386 & 0.386 & 4.272 & $0.02982^{* * *}$ & $<0.001$ \\
\hline Protein (\% of ration) & 1 & 0.1251 & 0.1251 & 1.385 & 0.00966 & 0.1744 \\
\hline Energy of ration (Kcal/lb AME) & 1 & 0.235 & 0.235 & 2.601 & $0.01816^{*}$ & 0.0165 \\
\hline Residuals & 77 & 6.9582 & 0.0904 & 0.53747 & & \\
\hline Total & 83 & 12.9461 & 1 & & & \\
\hline \multicolumn{7}{|l|}{ Weighted UniFrac distance } \\
\hline Days & 1 & 0.055783 & 0.055783 & 76.574 & $0.43079^{* * *}$ & $<0.001$ \\
\hline No. of parent flocks used & 1 & 0.001894 & 0.001894 & 2.6 & 0.01463 & 0.0509 \\
\hline Birds placed & 1 & 0.005532 & 0.005532 & 7.594 & $0.04272^{* * *}$ & $<0.001$ \\
\hline log CFU/g Campylobacter & 1 & 0.00484 & 0.00484 & 6.644 & $0.03738^{* * *}$ & $<0.001$ \\
\hline Protein (\% of ration) & 1 & 0.00092 & 0.00092 & 1.262 & 0.0071 & 0.2534 \\
\hline Energy of ration (Kcal/lb AME) & 1 & 0.004429 & 0.004429 & 6.08 & $0.0342^{* *}$ & $<0.01$ \\
\hline Residuals & 77 & 0.056093 & 0.000728 & 0.43318 & & \\
\hline Total & 83 & 0.129491 & 1 & & & \\
\hline
\end{tabular}

$p<0.1,{ }^{*} p<0.05,{ }^{* *} p<0.01,{ }^{* * *} p<0.001$

backgrounds represent whether predictors have a positive or a negative influence along with the significances, respectively, in the regression model. In addition, all categorical variables were 'dummyfied' (a standard procedure) to represent as present/absent as a tag and were used in the regression model to see whether their inclusion/exclusion has an effect on the final model. As expected, measuring alpha diversity, inclusion of day 30 samples increases richness and Shannon entropy. Although inclusion of day 7 samples led to an increased environmental pressure, it was marginally significant and therefore deterministic nature of microbial communities at local and terminal clade level should be taken with caution. In terms of how samples differ from each other, local contributions to beta diversity (LCBD) was also considered in subset regression analysis with positive/ red explanatory variables causing community structure to become different from the average community structure as a mean to identify groups that were markedly different. This was only observed for Bray-Curtis distance metric (that considers abundances of taxa without their phylogenetic distances) at day 7, and unweighted UniFrac (phylogenetic distance considering only presence/ absence of taxa without considering their abundance) for day 30 .

An increase in Protein_perc_ration led to a positive effect on microbial diversity, whilst simultaneously reducing the effect of environmental pressure on microbial community structure. In terms of beta diversity measure (LCBD with different distances), overall there was a reduction in beta diversity when considering Protein perc_ration, although the trend was opposite for unweighted UniFrac. Energy_of_ration also causes the microbial diversity to be more even as it had a positive 


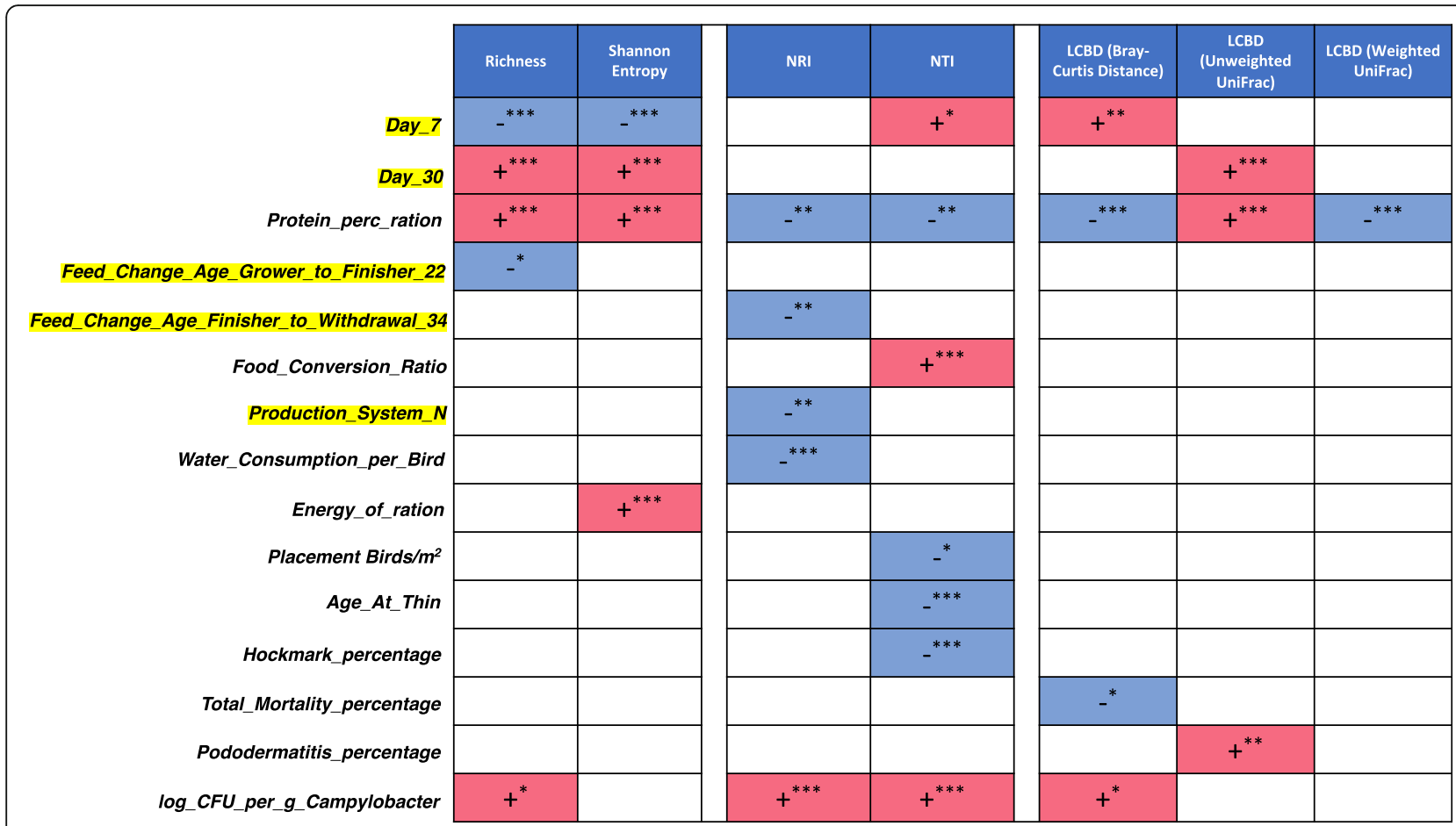

Fig. 3 Heatmap of key extrinsic parameters that influence different attributes of microbiome. The figure is based on subset regressions (Supplementary S4 to S10), where red and blue represent the significant positive and negative beta coefficients that were consistently selected in different regression models. The categorical variables are represented with a yellow highlight (coded as 1 (present) or 0 (absent)) and if selected is interpreted as the samples belonging to those categories having positive/negative influence on the respective microbiome metrics

and significant influence on Shannon entropy. The Feed_conversion_ratio on the other hand only caused shift in environmental pressure affecting the terminal clades by making them more clustered though the NTI measure.

In terms of production systems, it was observed that samples belonging to production system $\mathrm{N}$ had less influence by the environment and were possibly driven by competitive exclusion principles. Of note, production system $\mathrm{N}$ also had the lowest (best) Feed_conversion_ratio. A decrease in Feed_conversion_ratio was noted to lead to reduced environmental influence, which aligns with production system $\mathrm{N}$ having a reduced environmental influence, and also that a higher Placement_birds $/ \mathrm{m}^{2}$ resulting in a reduced environmental influence. The same phenomena were also observed when considering Age_at_thin, Hockmark_percentage and Water_consumption_per_bird as explanatory variables. Interestingly, recorded $\log _{-} \bar{C} F U_{-}$ per_g_Campylobacter led to an increase in microbial diversity, with an increase in environmental pressure (at global scale, NRI, and also at local terminal clustering, NTI) as well as causing a marked shift in terms of beta diversity. Of all production systems, Campylobacter was only identified in production system $\mathrm{N}$ at day 30 based on $16 \mathrm{~S}$ rRNA abundance count (Supplementary S2), corroborated with independent $\log \mathrm{CFU} / \mathrm{g}$ of Campylobacter measure.

\section{Discussion}

Our data clearly show that there is a difference in microbial community structure between production systems with varying influence by extrinsic parameters considered within this study. We observed diversity increase significantly for day 30 when compared to day 7 . This is in line with previous reports whereby the gastrointestinal (GI) tract of poultry comes into contact with exogenous microorganisms immediately after hatch and as the host grows, this microbiome becomes highly diverse until it reaches a relatively stable albeit dynamic state [25]. An important finding in this study is that amongst the different production systems, only $\mathrm{N}$ is where microbial community assemblage seems to be random (less environmental pressure). Stocking density is clearly a parameter which if varied, can alter microbial community structure significantly. This demonstrates that production systems can be modified to alter the microbiome profile influencing performance at farm level.

Dietary nutrient components are implicated in improving the performance of broiler chickens. An increasing Energy_of_ration and decreasing Protein_perc_ration in feed over time pertain to all production systems within this study. These variables have a direct influence on gut microbial composition, and in conjunction with differentiating parameters between production systems 
(i.e. stocking density), lead to differences in microbial composition between production systems. The role of diet is sufficiently important for microbial community structure assemblage as previously described whereby digestion of non-starch polysaccharides (NSPs; found in the grain of chicken feed) lead to production of SCFA, which are absorbed across mucosa and catabolised by the host $[15,26]$. SCFAs contribute to chicken nutrition and also lower $\mathrm{pH}$ which can inhibit acid-sensitive pathogens and improve mineral absorption $[17,18]$. Thus, an association exists between the consistent and differentiating parameters of production systems that affect feed utilisation, leading to competitive exclusion of genera based on competition for nutrients and other factors.

Genera that were differentially expressed between different production systems and days were identified (some also part of the core microbiome). For day 30, HW vs O comparison, Phascolarctobacterium, Thalassospira and Bacteroides were identified as increased for O. Phascolarctobacterium is involved in SCFA production, including acetate and propionate and described as an option for reduction of Campylobacter via competitive exclusion [27-29]. Here, Omega-3 fed poultry systems harboured Phascolarctobacterium at a higher prevalence, although we cannot state if this was directly associated with a reduction or absence of Campylobacters from the caecal community. Eisenbergiella was increased for HW vs $\mathrm{N}$ comparison at day 30, and Alistipes was increased for $\mathrm{N}$ when compared to $\mathrm{HW}$ at day 30 . A reduction in Eisenbergiella has been associated with gastrointestinal disorders linked to metabolic and microbiota changes (functional dyspepsia) resulting in defective energy metabolism, amino acids, nucleotides and SCFA [30]. The HW system may improve the metabolism-microbiome interaction and could result in a competitive exclusion of bacterial pathogens via a fortified immune system. A dysfunctional microbiota can induce metabolic, autoimmune and inflammatory diseases and can seriously undermine gut function [31-33]. Barnesiella was increased for HW when comparing to O. Presence of Barnesiella has been associated with prevention and spread of highly antibioticresistant bacteria [34] where the gut bacterial community may react to antibiotic inclusion in the diet and prophylactically encourage the presence of Barnesiella, an effect demonstrated in mice where ampicillin treatment increased their presence [35]. The competitive exclusion hypothesis is supported by the increase of Alistipes bacteria in $\mathrm{N}$ group at day 30 , and using the top- 25 most abundant taxa identified at OTU level where clear differences for $\mathrm{N}$ against $\mathrm{HW}$ and $\mathrm{O}$ were observed (also for genera Bacteroides). The presence of Subdoligranulum bacteria in HW and $\mathrm{O}$ production systems, although not discriminatory, still contributing to beta diversity, represents a sign of improved gut health as these bacteria are known to be involved in production of SCFAs (e.g. butyrate) with an important role in gut physiology [36]. Ruminiclostridium 5, identified within $\mathrm{HW}$ and $\mathrm{O}$ production systems, also within core microbiome, has been noted to impact SCFA concentration within the gut [37].

The European Union (EU) ban on antimicrobial growth promoters in 2006 has created an increased need to devise alternative methods to improve performance and potentially reduce numbers of pathogenic bacteria. Examples include use of natural plant-derived products such as carvacrol [38], addition of dietary prebiotics [39] and administration of live probiotic bacteria [40]. More recently, prebiotic galacto-oligosaccharides (GOS) have been added to broiler feed and enhanced the growth rate and feed conversion of chickens relative to those obtained with a calorie-matched control diet [41]. Recent developments have observed chicken diets enriched with Omega-3 PUFA's for benefits to human health [5]. Omega-3 fatty acids $\alpha$-linolenic acid (ALA) (often found in plants), eicosapentaenoic acid (EPA) and docosahexaenoic acid (DHA) (found typically in marine oils), all play a role in forming structural components of cell membranes, serve as precursors to bioactive lipid mediators and provide a source of energy [42]. PUFAs are also important as substrates for inflammatory and antiinflammatory acids, with EPA is believed to have antiinflammatory properties [43]. One of the proposed mechanisms as to how dietary antibiotics exert their growth promoting benefits is via anti-inflammatory effects towards the intestinal epithelium, by inhibition of production and excretion of catabolic mediators [44]. Increased levels of EPA following antibiotic supplementation align with this non-antibiotic, anti-inflammatory theory of antibiotic growth promotion [45]. In our study, we do not know if PUFAs were fully absorbed, or to what effect the interaction is with host microbiome; however, we do observe an increase in weight for production system $\mathrm{O}$ which has Omega-3. Of note, production system $\mathrm{N}$ had the lowest (best) FCR.

Remarkably, log_CFU_per_g_Campylobacter led to an increase in microbial diversity, an increase in environmental assemblage metrics NRI and NTI, and also increased divergence in community structure from other samples. Campylobacter was identified in production system $\mathrm{N}$ at day 30 (Supplementary S2) along with measuring the log CFU/g of Campylobacter corroborating the $16 \mathrm{~S}$ data. It has been previously reported that Campylobacter typically appears within week two of the life cycle $[11-14,46]$. The lack of identification of Campylobacter at any of the day 7 samples is anticipated. It is interesting that detection of Campylobacter was only observed at day 30 for production system $\mathrm{N}$. This may have been due to limitation of sampling points. Although we recognise that microbial diversity will increase naturally 
from day 7 to day 30, based on our data, Campylobacter is associated with an increasing diversity. Broiler genetics is known to impact microbial diversity $[47,48]$ and this may explain why in our study the environmental pressure was significantly impacted positively by the presence of Campylobacter. Here, the environmental influence may be the chicken host genetics influencing microbial community structure.

\section{Conclusion}

Our findings demonstrate a relative role of different production system parameters in shaping the bacterial communities' impact on the chicken microbiome, with stocking density playing a major role influencing microbial dynamics. Specific genera with higher prevalence were identified within production systems that have key roles in energy metabolism, amino acid, nucleotide and SCFA utilisation. It is clear that parameters between production systems (whether constant or variable) have an impact on microbial diversity which subsequently influences feed breakdown and hence instigates competitive exclusion of certain genera. Omega-3 had a positive impact on weight gain and Campylobacter presence was linked with environmental pressure, which could be either the external environment or the host itself. Future studies that will direct the optimisation of extrinsic parameters and optimisation of diets targeting microbes with the underlying benefits of improving performance will aid in reducing pathogens such as Campylobacter. Our study investigates the relative importance of production system parameters in an industrial farm environment without any intervention strategy (studying caecal microbiome at its natural environment), to reveal the factors that link microbial community structure to improved broiler performance and reduced pathogenic bacteria such as Campylobacter.

\section{Supplementary information}

Supplementary information accompanies this paper at https://doi.org/10 1186/s40168-020-00908-8.

Additional file 1: Supplementary S1-Subset Analysis.docx: Subset analysis from BVSTEP routine listing top subsets with highest correlation with the full genera table considering Bray-Curtis distance and considering samples provided in the first column. For each subset, PERMANOVA was performed against Groups considered in the first column.

Additional file 2: Supplementary S2-MINT Analysis.pdf: MINT studywise discriminant analysis between treatments ( $\mathrm{N}, \mathrm{HW}$, and $\mathrm{O})$. Additional methodology provided within file.

Additional file 3: Supplementary S3-Top 25 Abundant Taxa.pdf: Community structure based on relative abundance of the top-25 most abundant taxa identified at different taxonomic groups, where 'others' refers to all taxa not included in the 'top-25'.

Additional file 4: Supplementary S4-Richness Subset Regression.docx: Subset regression analysis using Richness as the dependent variable.
Additional file 5: Supplementary S5-Shannon Entropy Subset Regression.docx: Subset regression analysis using Shannon Entropy as the dependent variable.

Additional file 6: Supplementary S6-NRI Subset Regression.docx Subset regression analysis using phylogenetic alpha diversity (NRI) as the dependent variable.

Additional file 7: Supplementary S7-NTI Subset Regression.docx: Subset regression analysis using phylogenetic alpha diversity (NTI) as the dependent variable.

Additional file 8: Supplementary S8-LCBD Bray-Curtis Subset Regression.docx: Subset regression analysis using beta diversity measure (LCBD with Bray-Curtis distance) as the dependent variable.

Additional file 9: Supplementary S9-LCBD Unweighted UniFrac Subset Regression.docx: Subset regression analysis using beta diversity measure (LCBD with Unweighted UniFrac distance) as the dependent variable.

Additional file 10: Supplementary S10-LCBD Weighted UniFrac Subset Regression.docx: Subset regression analysis using beta diversity measure (LCBD with Weighted UniFrac distance) as the dependent variable.

Additional file 11: Supplementary S11-Pairwise Differential Analysis.xIsx: Differential analysis of genera that are up/down-regulated between different groups (Adjusted $P$ values $\leq 0.05$ ) with at least log2 fold change from the base mean abundances for the samples considered in the first column.

Additional file 12: Supplementary S12-

Bioinformatics_and_StatisticalAnalysis.docx: Bioinformatics and statistical analysis methods used within the study.

Additional file 13: Supplementary S13-Parameters Measured.docx: A list of all of the parameters measured within the study and their explanations.

\section{Abbreviations}

N: Normal; HW: Higher Welfare; O: Omega-3 Higher Welfare; SCFA: Short chain fatty acid; FCR: Feed conversion ratio; PUFA: Polyunsaturated fatty acids; AH: As hatched; gDNA: genomic DNA; OTUs: Operational taxonomic units; Gl: Gastrointestinal; NSPs: Non-starch polysaccharides; EU: European Union; GOS: Galacto-oligosaccharides; ALA: a-linolenic acid;

EPA: Eicosapentaenoic acid; DHA: Docosahexaenoic acid

Acknowledgements

Not applicable

Authors' contributions

AM, AR, UL, UZI, NC and OG contributed to the study design. NC and OG managed the study. CK, AM and ML performed the sample collection and DNA extraction. AM and OG performed the library preparation and Illumina MiSeq sequencing at the LSHTM. UZI wrote the analysis scripts to generate the figures and tables in this paper. UZI, AM and OG performed the bioinformatics and statistical analysis. UZI, AM, NC and OG drafted the initial version of the manuscript with all authors including BDG, ND, BWW and WTS contributing to redrafting. The authors read and approved the final manuscript.

Funding

OG and NC acknowledge research funding from Moy Park. UZI is funded by NERC Independent Research Fellowship (NE/L011956/1).

Availability of data and materials

The raw sequence files supporting the results of this article are available in the European Nucleotide Archive under the project accession number PRJEB34742.

Ethics approval and consent to participate

Not applicable

Consent for publication

Not applicable 


\section{Competing interests}

$A M, A R$ and $U L$ are employed by company Moy Park. AM is enrolled on a Ph.D. programme at QUB and undertook research work at AFBI and Moy Park. All other authors declare no competing interests.

\section{Author details}

'Moy Park, 39 Seagoe Industrial Estate, Portadown, Craigavon, Co. Armagh BT63 5QE, UK. ${ }^{2}$ School of Engineering, University of Glasgow, Glasgow G12 8LT, UK. ${ }^{3}$ Food Microbiology Unit, Agri-Food and Biosciences Institute, Newforge Lane, Belfast BT9 5PX, UK. ${ }^{4}$ Institute for Global Food Security, School of Biological Sciences, Queen's University Belfast, Biological Sciences Building, Belfast BT9 5DL, Northern Ireland. ${ }^{5}$ Faculty of Infectious \& Tropical Diseases, London School of Hygiene and Tropical Medicine, Keppel Street, London WC1E 7HT, UK.

Received: 20 May 2020 Accepted: 17 August 2020

Published online: 09 September 2020

\section{References}

1. Borda-Molina D, Seifert J, Camarinha-Silva A. Current perspectives of the chicken gastrointestinal tract and its microbiome. Computational and structural biotechnology journal. 2018;16:131-9.

2. FAO: Food outlook: biannual report on global food markets - November 2018. pp. 104: Rome; 2018:104.

3. Sood U, Gupta V, Kumar R, Lal S, Fawcett D, Rattan S, Poinern GEJ, Lal R. Chicken gut microbiome and human health: past scenarios, current perspectives, and futuristic applications. Indian J Microbiol. 2019:1-10.

4. Stanley D, Hughes RJ, Moore RJ. Microbiota of the chicken gastrointestinal tract: influence on health, productivity and disease. Appl Microbiol Biotechnol. 2014;98:4301-10.

5. Stanton AV, Shortall K, El-Sayed T, O'Donovan F, James K, Kennedy J, Hayes H, Fahey A, Dolan E, Williams D. Eating Omega-3 polyunsaturated fatty acid enriched chicken-meat and eggs results in increased plasma docosahexaenoic and eicosapentaenoic acid levels and an improved Omega-3-index. Circulation. 2017;136:A19913.

6. Amour C, Gratz J, Mduma E, Svensen E, Rogawski ET, McGrath M, Seidman JC, McCormick BJ, Shrestha S, Samie A, et al. Epidemiology and impact of campylobacter infection in children in 8 low-resource settings: results from the MAL-ED study. Clin Infect Dis. 2016:63:1171-9.

7. Hermans D, Pasmans F, Heyndrickx M, Van Immerseel F, Martel A, Van Deun $K$, Haesebrouck F. A tolerogenic mucosal immune response leads to persistent Campylobacter jejuni colonization in the chicken gut. Crit Rev Microbiol. 2012;38:17-29.

8. Humphrey S, Chaloner G, Kemmett K, Davidson N, Williams N, Kipar A, Humphrey T, Wigley P. Campylobacter jejuni is not merely a commensal in commercial broiler chickens and affects bird welfare. MBio. 2014;5:e01364-14

9. Van Deun K, Pasmans F, Ducatelle R, Flahou B, Vissenberg K, Martel A, Van den Broeck W, Van Immerseel F, Haesebrouck F. Colonization strategy of Campylobacter jejuni results in persistent infection of the chicken gut. Vet Microbiol. 2008;130:285-97.

10. Wigley P. Blurred lines: pathogens, commensals, and the healthy gut. Front Vet Sci. 2015;2:40.

11. Kalupahana RS, Kottawatta KS, Kanankege KS, van Bergen MA, Abeynayake P, Wagenaar JA. Colonization of Campylobacter spp. in broiler chickens and laying hens reared in tropical climates with low-biosecurity housing. Appl Environ Microbiol. 2013;79:393-5.

12. Neill SD, Campbell JN, Greene JA. Campylobacter species in broiler chickens. Avian Pathology. 1984;13:777-85.

13. Thibodeau A, Fravalo P, Yergeau E, Arsenault J, Lahaye L, Letellier A. Chicken caecal microbiome modifications induced by Campylobacter jejuni colonization and by a non-antibiotic feed additive. PLoS One. 2015;10: e0131978.

14. ljaz UZ, Sivaloganathan L, McKenna A, Richmond A, Kelly C, Linton M, Stratakos AC, Lavery U, Elmi A, Wren BW, et al. Comprehensive longitudinal microbiome analysis of the chicken cecum reveals a shift from competitive to environmental drivers and a window of opportunity for Campylobacter. Front Microbiol. 2018;9:2452.

15. Jozefiak D, Rutkowski A, Martin S. Carbohydrate fermentation in the avian ceca: a review. Anim Feed Sci Technol. 2004;113:1-15.
16. McNab JM. The avian caeca: a review. World's Poultry Science Journal. 2007; 29:251-63.

17. Sergeant MJ, Constantinidou C, Cogan TA, Bedford MR, Penn CW, Pallen MJ. Extensive microbial and functional diversity within the chicken cecal microbiome. PLoS One. 2014;9:e91941.

18. Apajalahti J. Comparative gut microflora, metabolic challenges, and potential opportunities. The Journal of Applied Poultry Research. 2005;14: 444-53.

19. Connerton PL, Richards PJ, Lafontaine GM, O'Kane PM, Ghaffar N, Cummings NJ, Smith DL, Fish NM, Connerton IF. The effect of the timing of exposure to Campylobacter jejuni on the gut microbiome and inflammatory responses of broiler chickens. Microbiome. 2018;6:88.

20. Huyghebaert $G$, Ducatelle R, Van Immerseel F. An update on alternatives to antimicrobial growth promoters for broilers. Vet J. 2011;187:182-8.

21. AL-Darkazali H, Meevootisom V, Isarangkul D, Wiyakrutta S. Gene expression and molecular characterization of a xylanase from chicken cecum metagenome. International journal of microbiology. 2017;2017.

22. D'Amore R, ljaz UZ, Schirmer M, Kenny JG, Gregory R, Darby AC, Shakya M, Podar M, Quince C, Hall N. A comprehensive benchmarking study of protocols and sequencing platforms for 16S rRNA community profiling. BMC Genomics. 2016:17:55

23. Klindworth A, Pruesse E, Schweer T, Peplies J, Quast C, Horn M, Glockner FO. Evaluation of general $16 \mathrm{~S}$ ribosomal RNA gene PCR primers for classical and next-generation sequencing-based diversity studies. Nucleic Acids Res. 2013:41:e1.

24. Clarke KR, Ainsworth M. A method of linking multivariate community structure to environmental variables. Mar Ecol Prog Ser. 1993;92:205-19.

25. Pan D, Yu Z. Intestinal microbiome of poultry and its interaction with host and diet. Gut Microbes. 2014;5:108-19.

26. McWhorter TJ, Caviedes-Vidal E, Karasov WH. The integration of digestion and osmoregulation in the avian gut. Biol Rev Camb Philos Soc. 2009;84: 533-65

27. Peralta-Sánchez JM, Martín-Platero AM, Ariza-Romero JJ, Zurita-González MJ, Baños A, Rodriguez-Ruano SM, Maqueda M, Valdivia E, Martínez-Bueno M, Rabelo-Ruiz M. Egg production in poultry farming is improved by probiotic bacteria. Front Microbiol. 2019;10:1042.

28. Wu F, Guo X, Zhang J, Zhang M, Ou Z, Peng Y. Phascolarctobacteriumáfaecium abundant colonization in human gastrointestinal tract. Experimental and therapeutic medicine. 2017;14: 3122-6.

29. Zheng M, Mao P, Tian X, Meng L. Growth performance, carcass characteristics, meat and egg quality, and intestinal microbiota in Beijingyou chicken on diets with inclusion of fresh chicory forage. Ital J Anim Sci. 2019;18:1310-20.

30. Luo L, Hu M, Li Y, Chen Y, Zhang S, Chen J, Wang Y, Lu B, Xie Z, Liao Q. Association between metabolic profile and microbiomic changes in rats with functional dyspepsia. RSC Adv. 2018:8:20166-81.

31. Ferreira CM, Vieira AT, Vinolo MAR, Oliveira FA, Curi R, Martins FdS: The central role of the gut microbiota in chronic inflammatory diseases. Journal of immunology research 2014, 2014.

32. Joyce SA, Gahan CG. The gut microbiota and the metabolic health of the host. Curr Opin Gastroenterol. 2014;30:120-7.

33. Silva MJB, Carneiro MBH, dos Anjos Pultz B, Pereira Silva D, Lopes MEdM, dos Santos LM: The multifaceted role of commensal microbiota in homeostasis and gastrointestinal diseases. Journal of immunology research 2015, 2015.

34. Ubeda C, Bucci V, Caballero S, Djukovic A, Toussaint NC, Equinda M, Lipuma $L$, Ling $L$, Gobourne A, No D, et al. Intestinal microbiota containing Barnesiella species cures vancomycin-resistant Enterococcus faecium colonization. Infect Immun. 2013;81:965-73.

35. O'Loughlin $J$, Samuelson DR, Braundmeier-Fleming AG, White BA, Haldorson GJ, Stone JB, Lessmann JJ, Eucker TP, Konkel ME. The intestinal microbiota influences Campylobacter jejuni colonization and extraintestinal dissemination in mice. Appl Environ Microbiol. 2015;81:4642-50.

36. Fleming SE, Fitch MD, DeVries S, Liu ML, Kight C. Nutrient utilization by cells isolated from rat jejunum, cecum and colon. J Nutr. 1991;121:869-78.

37. Song Y, Malmuthuge N, Steele MA, Guan LL. Shift of hindgut microbiota and microbial short chain fatty acids profiles in dairy calves from birth to pre-weaning. FEMS Microbiol Ecol. 2018;94:fix179.

38. Kelly C, Gundogdu O, Pircalabioru G, Cean A, Scates P, Linton M, Pinkerton $L$, Magowan E, Stef L, Simiz E, et al. The in vitro and in vivo effect of 
carvacrol in preventing campylobacter infection, colonization and in improving productivity of chicken broilers. Foodborne Pathog Dis. 2017;14: 341-9.

39. Sethiya NK. Review on natural growth promoters available for improving gut health of poultry: an alternative to antibiotic growth promoters. Asian Journal of Poultry Science. 2016;10:1-29.

40. Gadde U, Kim W, Oh S, Lillehoj HS. Alternatives to antibiotics for maximizing growth performance and feed efficiency in poultry: a review. Anim Health Res Rev. 2017;18:26-45.

41. Richards PJ, Lafontaine GMF, Connerton PL, Liang L, Asiani K, Fish NM, Connerton IF: Galacto-oligosaccharides modulate the juvenile gut microbiome and innate immunity to improve broiler chicken performance. mSystems 2020, 5 .

42. Jump DB, Tripathy S, Depner CM. fatty acid-regulated transcription factors in the liver. Annu Rev Nutr. 2013:33:249-69.

43. Calder PC. Omega-3 fatty acids and inflammatory processes. Nutrients. 2010; 2:355-74

44. Niewold TA. The nonantibiotic anti-inflammatory effect of antimicrobial growth promoters, the real mode of action? A hypothesis. Poult Sci. 2007; 86:605-9.

45. Gadde UD, Oh S, Lillehoj HS, Lillehoj EP. Antibiotic growth promoters virginiamycin and bacitracin methylene disalicylate alter the chicken intestinal metabolome. Sci Rep. 2018;8:3592.

46. Hermans D, Van Deun K, Martel A, Van Immerseel F, Messens W, HeyndrickX M, Haesebrouck F, Pasmans F. Colonization factors of Campylobacter jejuni in the chicken gut. Vet Res. 2011;42:82.

47. Psifidi A, Fife M, Howell J, Matika O, Van Diemen PM, Kuo R, Smith J, Hocking PM, Salmon N, Jones MA. The genomic architecture of resistance to Campylobacter jejuni intestinal colonisation in chickens. BMC Genomics. 2016;17:293.

48. Psifidi A, Kranis A, Rothwell L, Bremmer A, Russell K, Robledo D, Bush S, Fife M, Hocking P, Banos G: Genetic control of Campylobacter colonisation in broiler chickens: genomic and transcriptomic characterisation. bioRxiv 2020.

\section{Publisher's Note}

Springer Nature remains neutral with regard to jurisdictional claims in published maps and institutional affiliations.

Ready to submit your research? Choose BMC and benefit from:

- fast, convenient online submission

- thorough peer review by experienced researchers in your field

- rapid publication on acceptance

- support for research data, including large and complex data types

- gold Open Access which fosters wider collaboration and increased citations

- maximum visibility for your research: over $100 \mathrm{M}$ website views per year

At $\mathrm{BMC}$, research is always in progress.

Learn more biomedcentral.com/submissions 